\title{
Salud reproductiva femenina en el sur de la Amazonia colombiana
}

\section{Tania Yimara Martínez Forero}

Universidad Nacional de Colombia Sede Amazonia tymartinezf@unal.edu.co

\section{Lina Paola Garzón Garzón}

Universidad Nacional de Colombia Sede Amazonia lipgarzonga@unal.edu.co

\section{Carlos Franky Calvo}

Universidad Nacional de Colombia Sede Amazonia cefranky@unal.edu.co

RESUMEN

El objetivo del presente trabajo consiste en reconocer las prácticas de cuidado y atención de los agentes del sistema médico tradicional responsables de la salud reproductiva femenina en el sur de la Amazonia colombiana. Para ello, se realizaron entrevistas a profundidad, grupos focales y observación etnográfica con agentes locales de salud de cinco comunidades rurales y cascos urbanos de los municipios de Leticia y Puerto Nariño. Los resultados mostraron que la formación de personas, sus cuerpos y el buen trato, mediados por un constante control de las relaciones entre humanos y no humanos, se establecen como los determinantes de las prácticas de cuidado y atención a la salud reproductiva femenina. Igualmente, se evidencia un profundo respeto por las decisiones de la madre durante su proceso procreativo, lo que coincide con las recientes disposiciones internacionales. Estos datos sirven como base para la comprensión de la cultura médica local y su incorporación en los procesos terapéuticos de salud en un marco de interculturalidad.

Palabras clave: medicina tradicional, partería, plantas medicinales, región amazónica, salud materno-perinatal. 


\section{Female reproductive health in the south of the Colombian Amazon}

\section{ABSTRACT}

The purpose of this paper is to recognize the practices of care and attention of the agents of the traditional medical system responsible for female reproductive health in the south of the Colombian Amazon. In-depth interviews, focus groups and ethnographic observation with local health agents from five rural communities and urban areas of the Municipalities of Leticia and Puerto Nariño were conducted. The results showed that the formation of people, their bodies and good treatment, mediated by a constant control of the relationships between humans and non-humans and the search for good living, are established as the determinants of care practices and attention to women's reproductive health. Likewise, there is a deep respect for the mother's decisions during her procreative process, which coincides with the recent international provisions. These data serve as the basis for the understanding of the local medical culture and its incorporation into the therapeutic processes of health in a framework of interculturality.

Keywords: traditional medicine, midwifery, medicinal plants, Amazon region, maternal and perinatal health. 


\section{INTRODUCCIÓN}

Durante las últimas dos décadas se ha observado un elevado interés mundial por visibilizar las condiciones de salud de la mujer durante su fase fértil y procreativa, dado que su cuidado y atención son «una obligación de la sociedad, que contribuye a elevar el desarrollo social y humano de las actuales y futuras generaciones» (Ministerio de Salud y Protección Social - MSPS y Fondo de Población de las Naciones Unidas - UNFPA, 2014, p. 15). Si bien Colombia presenta un gran avance en la atención a la salud reproductiva en establecimientos y por cuenta de personal calificado — que viene en aumento desde 1990—, esto no refleja la realidad nacional.

En la Amazonia, diversas condiciones adversas conducen no solo a una falta de atención médica (Profamilia et al., 2011, p. 207) en las zonas alejadas, sino la obligatoria remisión de pacientes desde los centros de primer nivel hacia la capital departamental (Leticia) o nacional (Bogotá) (Campos, 2001, p. 175). Dentro de estas se encuentran las condiciones geográficas, población rural dispersa $\left(1,3\right.$ habitantes $\left./ \mathrm{km}^{2}\right)$, falta de medios de transporte terrestre, altos costos para la movilidad y estadía, ausencia de centros hospitalarios de segundo y tercer nivel en áreas rurales, y falta de equipamiento y programas en salud dirigidos a las comunidades indígenas, en especial a las más alejadas.

En ese sentido, el Amazonas se establece hoy en día como el departamento con menor atención al parto en hospitales o centros de salud $(68,8 \%)$, pues un tercio de las mujeres $(31,1 \%)$ vive el proceso de alumbramiento en su lugar de residencia (MSPS, 2013, p. 229; MSPS, 2014, p. 48; Profamilia et al., 2011, p. 221). Igualmente se establece como una de las regiones con menor proporción de atención prenatal por médico (Profamilia et al., 2011).

Esta baja atención médica diplomada a las etapas reproductivas no es solo un producto de variables geográficas y económicas, sino también de la difícil comu- 
nicación entre las instituciones y la población. Esto se debe a relación asimétrica entre médicos y pacientes y a la falta de legitimación de las prácticas culturales, por el desconocimiento de la visión colectiva frente a la salud reproductiva que tienen los pueblos amazónicos. No obstante, hay una evidente credibilidad social que la población imprime sobre el Sistema Biomédico - SBM — para el manejo de enfermedades, control de la natalidad y medicación para el dolor asociado al parto (Reyes, 2009a, pp. 101 y 128).

La reducida presencia rural de centros de salud ha redundado en la evidente predominancia del sistema médico tradicional —SMT-, que por su cercanía geográfica y cultural cuenta con una mayor asistencia para la población indígena. Este sistema corresponde, de manera general, a un enfoque del «buen vivir» presente en las etnias amazónicas, que integra varias esferas de la vida social, tales como alimentación, ritos, cuidados y relaciones sociales, que definen a la persona dentro de un cuerpo colectivo. Por ende, las nociones de salud y enfermedad asociadas a la salud reproductiva femenina no son vistas como procesos alejados de la formación física y moral de las personas dentro de la comunidad. Por lo contrario, se encuentran asociadas a una diversidad de pautas conductuales y restricciones que demuestran no solo un gran conocimiento de las especies animales y vegetales con las que conviven, sino también la búsqueda constante del mantenimiento de la fertilidad en el territorio como garante de la pervivencia de estos pueblos.

En ese sentido, el presente trabajo se orientó a reconocer las prácticas de atención, cuidado y medicina vegetal relacionada con la salud reproductiva femenina en el sur del Amazonas, que mantienen los diferentes agentes del SMT como un aporte para la futura implementación de programas de interculturalidad en salud que integren las nociones de salud y enfermedad asociadas a la formación física y moral de las personas dentro de la comunidad. Estas nociones establecen pautas conductuales, curaciones y restricciones que demuestran no solo un gran conocimiento de las especies animales y vegetales con las que conviven, sino también la búsqueda constante del mantenimiento de la fertilidad en el territorio como garante de la pervivencia de estos pueblos.

\section{MATERIALES Y MÉTODOS}

El estudio se realizó con población indígena y agentes de salud comunitarios (parteras, médicos tradicionales, chamanes y auxiliares de salud pública) y profesionales (médicos y enfermeros) residentes en las comunidades Km. 11 
$\mathrm{N}+$ maira na+mek+ibur+, San Martín de Amacayacu, Resguardo TICOYA, San Juan del Socó, San Juan de Atacuari y casco urbano de los municipios de Leticia y Puerto Nariño, cuya característica común fue el autoidentificarse como indígenas tikuna, yagua, cocama, bora o murui o ser agente responsable de la salud reproductiva femenina.

La muestra poblacional fue seleccionada mediante muestreo por conveniencia y bola de nieve, es decir, conformada por sujetos voluntarios o casuales, que permitieron convocar a otros agentes relacionados (Patton, 2002, p. 42). Ya establecidos los contactos, se realizaron entrevistas a profundidad con los agentes comunitarios y profesionales encargados de la salud reproductiva de las mujeres y grupos de discusión con mujeres, madres y abuelas de las comunidades periurbana y urbana, además de observación etnográfica por medio de la participación en actividades cotidianas como la chagra, el procesamiento de las comidas, juegos, aseo, cuidados, etc. La información fue analizada a través de una estrategia de categorización y triangulación de los datos sistematizados en el programa AtlasTi 7.0.

\section{RESULTADOS}

En el marco de la salud reproductiva femenina, el SMT organiza sus agentes de manera jerarquizada según la dolencia y tipo de intervención (Patiño y Sandín, 2014, p. 380). Existe un primer nivel donde se desarrollan las prácticas de cuidado y autocuidado en el hogar (dolencias como cólicos, infecciones urinarias, anticoncepción) y la formación de la persona en sí; un segundo nivel conformado por las parteras encargadas de la atención a las etapas procreativas; un tercer nivel atendido por médicos tradicionales cuyos conocimientos en etnobotánica y dominio espiritual les permiten atender complicaciones asociadas al parto y al nacimiento, y finalmente, un cuarto nivel o especializado, que presenta al chamán como entidad máxima, quien se constituye como el intermediario entre los humanos y la esfera espiritual, encargado principalmente del amarre o desamarre de la matriz para el control de la fecundidad y de las dolencias más complejas asociadas al aparato reproductivo femenino.

Estos agentes comunitarios desarrollan sus prácticas de atención y cuidados durante cuatro etapas del ciclo de vida (fertilidad, embarazo, parto y dieta), cuyas subetapas responden a eventos biológicos evidentes (pérdida de la menstruación, vientre protuberante, salida de los dientes, etc.), más que a períodos de tiempo o de edad específicos. 


\section{FERTILIDAD}

Esta etapa, conocida como preconcepcional, se divide en dos subetapas: la infancia y la primera menstruación o menarquia/conformación de pareja. Desde la primera de estas subetapas se imparten los cursos de «preparación para la maternidad y la paternidad» (o «psicoprofilácticos» ofrecidos por el SBM durante la gestación). Durante los primeros años de vida, niños y niñas son formados por medio del juego en terrenos propensos a raspones, cortadas, etc., y la participación directa en las labores cotidianas del hogar, como el cuidado de los más pequeños y las labores propias de la chagra, que conllevan a la formación de cuerpos fuertes, resistentes, con gran aceptación del dolor y de la transición a la maternidad y paternidad. Igualmente, se desarrollan prácticas de curación, como el consumo de una cucharada de aceite de raya negra (Potamotrygon sp.) aproximadamente a los nueve años, para que la niña en su adultez pueda parir fácil.

Durante la segunda subetapa, se evidencian prácticas rituales y de curación para la fertilidad, como por ejemplo los rezos chamanísticos en el marco de la pelazón tikuna ${ }^{1}$, ya sea para retardar el proceso de menarquia por medio de la tabaqueada durante la primera perforación de orejas en las niñas - infancia-, o para amarrar el vientre con un hilo espiritual para retardar el proceso de fecundidad - menarquia — . Cuando ya se establece la pareja, también se recurre a métodos anticonceptivos alopáticos ofrecidos gratuitamente por las Instituciones Prestadoras de Salud - IPS - o al uso de medicina vegetal como la toma de palo sangre (Brosimum rubescens) durante los días del período menstrual (ver tabla 1).

A menudo esta decisión no cuenta con una valoración positiva en la comunidad, por lo que se tiene que ocultar a las parejas la utilización de métodos anticonceptivos alopáticos, tal como explica un auxiliar de salud pública tikuna residente en el área rural:

La planificación familiar es un problema para el tikuna, porque no es de su cultura, pero de poco a poco con la educación que le estamos dando hay madres que sí se están acogiendo a eso.

1 «La fiesta de la pelazón ( $y u$-u-echíga) es el rito de iniciación de la mujer tikuna que se realiza cuando ocurre la primera menstruación o menarquia. [...] La menstruación convierte a la niña de cuerpo infantil en una mujer madura, y por esta razón, la joven debe prepararse y recibir los consejos de su familia para asumir y enfrentar esta transición. La joven en esta etapa es llamada en lengua tikuna: wôrekü» (Reyes, 2009a, p. 149). 
Tabla 1. Plantas medicinales para la anticoncepción

\begin{tabular}{llllll}
\hline $\begin{array}{l}\text { Nombre } \\
\text { común }\end{array}$ & $\begin{array}{l}\text { Nombre } \\
\text { científico }\end{array}$ & $\begin{array}{l}\text { Parte } \\
\text { utilizada }\end{array}$ & Preparación & $\begin{array}{l}\text { Adminis- } \\
\text { tración }\end{array}$ & Dosificación \\
\hline $\begin{array}{l}\text { Palo sangre } \\
\text { Brosimum } \\
\text { rubescens }\end{array}$ & Corteza & Decocción & Oral & $\begin{array}{l}\text { Varias tomas al día hasta } \\
\text { acabar el preparado }\end{array}$ \\
\hline Achiote & Bixa orellana & Fruto & Decocción & Oral & Una toma \\
\hline Mururé & $\begin{array}{l}\text { Bronsinum } \\
\text { Acotifolin }\end{array}$ & Corteza & Decocción & Oral & Varias tomas al día hasta \\
acabar el preparado
\end{tabular}

Los cambios culturales relacionados con las responsabilidades domésticas, producto de las actuales cargas laborales, menor participación del hombre en la crianza, madresolterismo, violencia intrafamiliar - generalmente causada por el consumo de alcohol-, ingreso al sistema educativo, entre otras, son variables sociales que han estimulado en las mujeres un mayor control de la natalidad. Por ello, en el caso de embarazos no deseados, ocasionalmente se recurre a métodos caseros inseguros que combinan medicina alopática y vegetal, tal como explica una partera tikuna residente en área rural: 
Ahora hay una moda entre las jóvenes, cuando quedan embarazadas y no quieren, se toman un agua de limón con alka seltzer y una pastilla que no sé qué será, pues, para sacarlo. Yo no estoy de acuerdo, porque eso puede dañar su salud.

Cabe resaltar el profundo conocimiento que tienen los pueblos amazónicos frente a la medicina vegetal destinada a la interrupción voluntaria del embarazo (ver tabla 2), que, debido a la incorporación de los principios religiosos en la mayoría de las comunidades, hoy en día forman parte de las recomendaciones dictadas por los agentes comunitarios de salud, como contraindicaciones durante la gestación.

Tabla 2. Plantas medicinales con efectos emenagogos o abortivos

\begin{tabular}{|c|c|c|c|c|c|}
\hline $\begin{array}{l}\text { Nombre } \\
\text { común }\end{array}$ & $\begin{array}{l}\text { Nombre } \\
\text { científico }\end{array}$ & $\begin{array}{l}\text { Parte } \\
\text { utilizada }\end{array}$ & Preparación & $\begin{array}{l}\text { Adminis- } \\
\text { tración }\end{array}$ & Dosificación \\
\hline Limón & $\begin{array}{l}\text { Citrus limon (L.) } \\
\text { Osbeck }\end{array}$ & Fruto & $\begin{array}{l}\text { No se procesa/ } \\
\text { Mezcla con sal }\end{array}$ & Oral & $\begin{array}{l}\text { Tomar varias veces } \\
\text { durante un día }\end{array}$ \\
\hline \multirow[t]{2}{*}{ Piña } & \multirow{2}{*}{$\begin{array}{l}\text { Ananas } \\
\text { ananassoides }\end{array}$} & Bulbo & Decocción & Oral & Una toma \\
\hline & & $\begin{array}{l}\text { Fruto } \\
\text { inmaduro }\end{array}$ & No se procesa & Oral & $\begin{array}{l}\text { Tomar varias veces } \\
\text { durante un día }\end{array}$ \\
\hline Piri Piri & $\begin{array}{l}\text { Eleutherine } \\
\text { bulbosa }\end{array}$ & Hoja & Decocción & Oral & Una toma \\
\hline Jengibre & $\begin{array}{l}\text { Zingiber } \\
\text { officinale Roscoe }\end{array}$ & Rizoma & Decocción & Oral & Una toma \\
\hline Aguacate & $\begin{array}{l}\text { Persea } \\
\text { americana }\end{array}$ & Semilla & $\begin{array}{l}\text { Mezcla con } \\
\text { alcohol }\end{array}$ & Oral & Una toma \\
\hline Tumbo & $\begin{array}{l}\text { Passiflora } \\
\text { tripartita }\end{array}$ & Hoja & Decocción & Oral & Una toma \\
\hline $\begin{array}{l}\text { Huacapu- } \\
\text { rana }\end{array}$ & $\begin{array}{l}\text { Campsiandra } \\
\text { angustifolia }\end{array}$ & Corteza & Decocción & Oral & $\begin{array}{l}\text { Tomar dos veces } \\
\text { durante un día }\end{array}$ \\
\hline Asaí & Euterpe oleracea & Raíz & Decocción & Oral & $\begin{array}{l}\text { Tomar dos o tres } \\
\text { veces durante un } \\
\text { día }\end{array}$ \\
\hline $\begin{array}{l}\text { Palo } \\
\text { sangre }\end{array}$ & $\begin{array}{l}\text { Brosimum } \\
\text { rubescens }\end{array}$ & Corteza & Decocción & Oral & $\begin{array}{l}\text { Tomar dos veces } \\
\text { durante un día }\end{array}$ \\
\hline
\end{tabular}

Frente a un embarazo no deseado - que suele ocurrir tras un número considerable de hijos—, la mujer asume con evidente resignación la llegada de un 
nuevo hijo, aspecto que genera una ruptura en el establecimiento del vínculo afectivo necesario para el cuidado del neonato y la protección de la salud física y mental de madre. Ello ha venido generando un impacto negativo, dado que el vínculo madre-hijo/a-padre y la red de apoyo extendido que lo protege se han consolidado históricamente en los pueblos amazónicos como el bastión principal que soporta el «buen vivir» — aspecto que se desvanece frente a la vivencia de una maternidad no deseada_-, tal como relata una joven tikuna residente en el área rural:

Acá hubo un caso de una mujer que permanecía siempre triste, con cada hijo triste. Ella demandó al marido porque le pega y no hacía más que tener y tener hijos y ella ya no quería más, pero el marido no la deja planificar. Acá los hombres ven mal eso: si una mujer planifica ya dicen que está con otro, que es de esas. Entonces la mujer, cuando demandó, creo que le pusieron la pila en el hospital porque así es como planificamos nosotras, con la pila en el brazo [implante subcutáneo anticonceptivo Jadelle].

Sin embargo, ante la conformación de la pareja, es común el deseo de procreación. Para ello se recurre a tratamientos, generalmente vegetales, para la consecución del embarazo (ver tabla 3 ).

Tabla 3. Plantas medicinales promotoras de embarazo

\begin{tabular}{llllll}
\hline $\begin{array}{l}\text { Nombre } \\
\text { común }\end{array}$ & $\begin{array}{l}\text { Nombre } \\
\text { científico }\end{array}$ & $\begin{array}{l}\text { Parte } \\
\text { utilizada }\end{array}$ & Preparación & $\begin{array}{l}\text { Adminis- } \\
\text { tración }\end{array}$ & Dosificación \\
\hline Aguacate & $\begin{array}{l}\text { Persea } \\
\text { americana }\end{array}$ & Semilla & $\begin{array}{l}\text { Mezcla con alcohol } \\
\text { y colmena de abeja }\end{array}$ & Oral & $\begin{array}{l}\text { Tomar por siete } \\
\text { días en la mañana }\end{array}$ \\
\hline $\begin{array}{l}\text { Algodón } \\
\text { morado }\end{array}$ & $\begin{array}{l}\text { Gossypium } \\
\text { herbaceum L. }\end{array}$ & Raíz & Decocción & Oral & $\begin{array}{l}\text { Tomar por siete } \\
\text { días en la mañana }\end{array}$ \\
\hline Naranja & $\begin{array}{l}\text { Citrus } \\
\text { sinensis }\end{array}$ & Raíz & Decocción & Oral & $\begin{array}{l}\text { Tomar por siete } \\
\text { días en la mañana }\end{array}$ \\
\hline $\begin{array}{l}\text { Renaco/ } \\
\text { renaquillo }\end{array}$ & $\begin{array}{l}\text { Clusia } \\
\text { rosea }\end{array}$ & Hoja & Decocción/ Mezcla & Oral & $\begin{array}{l}\text { Tomar por cinco } \\
\text { días }\end{array}$ \\
\hline Cortadera & $\begin{array}{l}\text { Scleria } \\
\text { secans }\end{array}$ & Hoja & Decocción/ Mezcla \\
con otras plantas & Oral & $\begin{array}{l}\text { Toma por cinco } \\
\text { días }\end{array}$ \\
\hline Piñón & $\begin{array}{l}\text { Jatropha } \\
\text { curcas }\end{array}$ & Raíz & Decocción & Oral & $\begin{array}{l}\text { Tomar por siete } \\
\text { días en la mañana }\end{array}$ \\
\hline Asaí & $\begin{array}{l}\text { Euterpe } \\
\text { oleracea }\end{array}$ & Raíz & Decocción & Oral & $\begin{array}{l}\text { Tomar por siete } \\
\text { días en la mañana }\end{array}$ \\
\hline
\end{tabular}




\section{EMBARAZO}

Esta etapa se encuentra conformada por tres subetapas: (i) «criatura en formación»; (ii) «criatura formadita», y (iii) «preparación para el parto», que coinciden generalmente con las establecidas por el SBM por sus objetivos, mas no por sus rangos de tiempo.

Durante los primeros meses se presenta la primera etapa llamada «criatura en formación», que inicia cuando la mujer se da cuenta del embarazo, ya sea por la pérdida de la menstruación, por el canto del gavilán popoví, cocorí o rabicorto (Buteo brachyurus) y del búho urukutú, murucutú o guardacaminos negruzco (Hidropsalis nigrescens), así como la vivencia de los síntomas asociados a la preñez o por la salida de un vientre protuberante.

En este estadio se pueden observar prácticas para el cambio de sexo mediante el consumo de especies vegetales como el aguaje (Mauritia flexuosa) y la caña de azúcar (Saccharum officinarum) para que sea mujer, o de animales como el agua de pene de taricaya (Podocnemis unifilis) para que sea hombre. Asimismo, se siembra la placenta durante el posparto inmediato, de tal manera que se coloca hacia arriba si se desea un niño o al contrario si se quiere una niña. Estas prácticas también han sido reportadas por Rivas (2015) con población tikuna, cocama y yagua en la Amazonia peruana.

Durante esta etapa suelen ser comunes las amenazas de aborto, ya sea por condiciones físicas o por accidentes propios de las actividades de chagra o domésticas. Para ello, los médicos tradicionales o chamanes hacen curaciones con el fin de que el embrión se mantenga adherido a la matriz. Por ejemplo, acuden a la invocación mediante rezos de especies vegetales como el caimo (Crysophyllum cainito) debido a sus características pegajosas. También recurren a especies animales como la avispa (Pepsis sp.), cuya cría cuelga del hogar arcilloso que hace para ella o al ave ayaymama (Nyctibius aetherus), que al no hacer nido, coloca su huevo en la rama superior de un árbol del que nunca cae.

En la segunda etapa, cuando está la criatura «formadita» y ya se siente, empieza todo el proceso de asesoría o control prenatal. Dicho control se encuentra bajo la responsabilidad de las abuelas, madres, parteras o parteros, aspecto que depende de la residencia, afinidades y nociones culturales propios de la etnia de la madre y el padre, y cuya frecuencia varía según el agente de salud escogido.

De igual manera, se dictan las dietas que seguirá la madre durante el transcurso de su embarazo. Estas dietas se caracterizan por la restricción del consumo de 
especies animales y vegetales que puedan cutipar ${ }^{2}$ al bebé, otorgándole características indeseables, o a la madre generando complicaciones durante el parto. Un ejemplo de ello es la prohibición de los animales encuevados que trancan al bebé y no permiten que el parto avance, como la cucha (Hypostomus plecostomus), el armadillo (Dasypus novemcinctus) o el morrocoy (Chelonoidis carbonaria). También se restringe el consumo de animales pintados, como la capibara (Hydrochoerus hydrochaeris), debido a que el bebé nace manchado, y de animales nocturnos, como el mono cusumbo (Potos flavus) o el mico nocturno (Aotus nancymaae), porque al recién nacido se le dificultará dormir. Además, la madre debe evitar acercarse a la lupuna ${ }^{3}$ (Ceiba pentandra) porque puede generar malformaciones o problemas congénitos en el neonato conocidos como «mal de lupuna».

Para la tercera etapa de «preparación para el parto», los esfuerzos se concentran en obtener una disposición favorable del bebé para un parto vaginal. Para ello se recurre a la «sobada», ya sea de la partera o por la misma madre. En caso de encontrar que la criatura se encuentra de pies o nalgas (feto en presentación podálica), los médicos tradicionales o chamanes atraerán las propiedades de especies animales como la ardilla (Sciurus igniventris) o la lagartija (Podarcis sp.), cuya posición para bajar zonas verticales es siempre cabeza abajo, característica que busca ser incorporada en el cuerpo del feto por medio de la invocación.

También se aconseja a la madre tomar agua de río y escupirla en dirección a la corriente, para que el parto fluya con completa naturalidad. Finalmente, se restringen los alimentos grasosos (como peces de cuero) y con altos contenidos de glucosa, para evitar que el bebé engorde y, así, se dificulte su salida.

\section{PARTO}

Esta etapa se encuentra conformada por cuatro subetapas: (i) la «seña»; (ii) «entrar en dolores», que finaliza cuando a la parturienta «le llega la hora»; (iii) «hacer fuerza», y (iv) «descansar». Estas subetapas, en palabras del SBM, se asemejan a

2 Las cutipas son enfermedades o rasgos no deseados que se incorporan al cuerpo del feto, neonato o madre, por el contacto directo o indirecto con animales, plantas e incluso comportamientos o trabajos caracterizados por dichos rasgos. Su origen reside en la infracción a las normas sociales e incumplimiento de las buenas costumbres, por lo que su sanación radica en la reconciliación con el agente causante (Ministerio de Salud del Perú, 2006, p. 207).

3 Se dice que la lupuna o ceiba es uno de los 123 árboles del Amazonas que tienen madre. Por ello, la evasión de su contacto se da incluso antes del embarazo, durante la menstruación y en el momento del parto, pues en cualquiera de los casos genera malformaciones en el neonato. 
(i) preparto o prodrómico; (ii) «parto activo»; (iii) «expulsivo», y (iv) «nacimiento y alumbramiento», respectivamente.

Durante la primera etapa, es decir la «seña» o preparto, se presentan los «signos prodromales»: el conjunto de síntomas que permiten reconocer que el parto se acerca, como por ejemplo la expulsión del tapón mucoso, presión en pelvis y recto, contracciones preparatorias o de Braxton Hicks, instinto de anidamiento ${ }^{4}$, malestares estomacales, entre otras. Dadas sus variaciones y la continuidad en las actividades cotidianas de la mujer hasta que el cuerpo lo permite — comúnmente hasta el inicio de un parto latente, e incluso activo-, muchas de las mujeres no se dan cuenta de esta etapa y por ello no la nombran.

En la segunda etapa, o «entrar en dolores», la mujer experimenta contracciones irregulares que aumentan significativamente conforme avanza el trabajo de parto. Es en este punto donde parteras, madres, abuelas o ellas mismas alistan los elementos necesarios, dependiendo del lugar donde se dará el parto. Para un parto institucional se tiene lista la maleta con la ropa de madre y bebé, pañales y elementos de aseo, y si el parto es en la casa o en el monte, se alistan los elementos para el corte y ligadura de cordón (isana ${ }^{5}$ e hilo negro respectivamente), con el fin de adecuar el lugar de parto para la intimidad y el calor (con hojas de plátano y mantas), así como las medicinas vegetales o mantecas, para «deslizar» y «botar el frío».

Dentro de las medicinas vegetales y productos animales más utilizados en el parto se encuentran la malva (Malva silvestris) y pelusa de lulo (Solanum quitoense) para deslizar (ver tabla 4), por ser flemosas o resbalosas, y las mantecas de gallina negra (Gallus gallus) y raya (Potamotrygon sp.), por ser animales que paren fácil. Para «botar el frío», el algodón morado (Gossypium herbaceum L.) y la corteza de ubo (Spondias mombin).

El algodón morado (Gossypium herbaceum L.) es quizá la especie vegetal más utilizada en la Amazonia colombiana, dadas sus propiedades oxitócicas que fomentan las contracciones. No obstante, entre la comunidad médica no cuenta con gran aprobación, pues la aceleración espontánea de la contractibilidad uterina resulta nociva para un útero y cuello uterino que no se encuentren en las condiciones óptimas de dilatación y borramiento (medidos a través del índice de Bishop por

$4 \quad$ Al parecer, así como ocurre en diferentes especies del reino animal, muchas gestantes experimentan un fuerte impulso por preparar el espacio para la llegada de sus hijos. Dicho impulso se ve acompañado por un comportamiento - casi impulsivo - por organizar el entorno y generar un espacio confortable y limpio, un evidente aumento de la energía y vitalidad, además de altas dosis de ansiedad por la llegada del nuevo ser al hogar.

5 Extraída de la cañabrava o caña flecha (Gynerium sagittatum). 
Tabla 4. Plantas medicinales para el parto

\begin{tabular}{|c|c|c|c|c|c|}
\hline $\begin{array}{l}\text { Nombre } \\
\text { común }\end{array}$ & $\begin{array}{l}\text { Nombre } \\
\text { científico }\end{array}$ & $\begin{array}{l}\text { Parte } \\
\text { utilizada }\end{array}$ & Preparación & $\begin{array}{l}\text { Adminis- } \\
\text { tración }\end{array}$ & Dosificación \\
\hline Malva & Malva silvestris & Hoja & $\begin{array}{l}\text { Maceración/ } \\
\text { decocción }\end{array}$ & $\begin{array}{l}\text { Oral/ } \\
\text { tópico }\end{array}$ & $\begin{array}{l}\text { Varias tomas } \\
\text { en un día }\end{array}$ \\
\hline $\begin{array}{l}\text { Algodón } \\
\text { morado }\end{array}$ & $\begin{array}{l}\text { Gossypium } \\
\text { herbaceum L. }\end{array}$ & Hoja & $\begin{array}{l}\text { Decocción/ } \\
\text { Mezcla con } \\
\text { otras plantas }\end{array}$ & $\begin{array}{l}\text { Oral/ } \\
\text { baño }\end{array}$ & Una toma \\
\hline Cacao & $\begin{array}{l}\text { Theobroma } \\
\text { cacao }\end{array}$ & $\begin{array}{l}\text { Fruto/ } \\
\text { corteza }\end{array}$ & $\begin{array}{l}\text { Decocción/ } \\
\text { Mezcla con } \\
\text { otras plantas }\end{array}$ & Baño & Un baño \\
\hline $\begin{array}{l}\text { Itininga/ } \\
\text { tripa de } \\
\text { gallina } \\
\end{array}$ & $\begin{array}{l}\text { Philodendron } \\
\text { camposportua- } \\
\text { num }\end{array}$ & Hoja & Decocción & Oral & Una toma \\
\hline Lulo & $\begin{array}{l}\text { Solanum } \\
\text { quitoense }\end{array}$ & Fruto & No se procesa & Oral & Una toma \\
\hline Santamaría & Piper peltatum & Bulbo & $\begin{array}{l}\text { Maceración/ } \\
\text { Decocción }\end{array}$ & Oral & $\begin{array}{l}\text { Varias tomas } \\
\text { en un día }\end{array}$ \\
\hline
\end{tabular}

el SMT). Esto puede repercutir de manera nociva tanto en la evolución del parto como en el estado de alerta del recién nacido, tal como es explicado por la médica encargada de los servicios gineco-obstétricos en una entidad del casco urbano:

Realmente sí acelera el trabajo de parto, pero los niños me he dado cuenta de que nacen deprimidos, son niños que nacen muy atónicos y hay que estarlos estimulando mucho más que al resto de niños que no han tomado este tipo de aguas. Yo me he dado cuenta porque yo les pregunto [...], y es que lo vemos en la monitoría se encuentra una taquicardia fetal que de repente aparece y luego ya se calma [...], incluso algunos nacen meconiados.

Por otra parte, la falta de empatía con la parturienta, así como su desconfianza y miedo durante el trabajo de parto, es visto como un determinante de complicaciones que debe ser prevenido por medio de palabras suaves, familiares y buen trato o «trato bonito». Este trato responde igualmente a una noción cultural en la que el estado emocional de la madre y sus acompañantes se establece como uno de los determinantes del temperamento e identidad del neonato. Ello es evidente en tres relatos: los dos primeros de un hombre y una mujer tikuna, residentes en el área rural, y el tercero, de una abuela murui residente en el área periurbana: 
Hay que saberlo tratar, porque cualquier maltrato es complicación, porque ellas están delicadas.

Acá hubo una complicación [...] por negligencia [de la mamá], porque ya no quería que la tocaran, tenía mucho miedo, no tenía confianza [...].

Pa' que nazca bonito, hay que sobarle y hablarle bonito.

Esta segunda etapa culmina cuando se da la dilatación completa, es decir, cuando le «llega la hora». Dicha dilatación es valorada por el agente de salud acompañante, quien toca la corona de la madre, que va calentándose en un círculo que progresivamente se cierra conforme avanza la apertura y borramiento de su cérvix. Cuando la corona se encuentra completamente caliente, significa que ya debe «hacer la fuerza».

Durante la tercera etapa, es decir, «hacer la fuerza» o expulsivo, la mujer debe asumir la posición más cómoda (generalmente en cuclillas), ya sea por decisión propia o por orientación de la partera. Ello también dependerá de los acompañantes y recursos disponibles que puedan servir de soporte. Si cuenta con la compañía del marido, este podrá sostenerla por sus brazos desde atrás, o recurriendo a elementos del entorno que puedan servir de soporte (por ejemplo, hamaca, palo, butacas, etc.). Dado que el parto en los domicilios más apartados suele ser a solas, las abuelas son las encargadas de enseñar con anticipación las posiciones y recursos a los que la madre puede acceder para realizar el «pujo».

En caso de complicaciones, se llama al médico tradicional o chamán, quien se encarga de hacer los rezos, curaciones o «destrancar» a la madre cuando el expulsivo está muy prolongado. En el primero de los casos, los rezos y curaciones dependen del tipo de complicación y su origen. Si el origen se da por la cutipa de algo consumido durante el embarazo, se recurre a la incorporación de las cualidades opuestas mediante la invocación de las características deslizantes de especies vegetales que resbalan, como por ejemplo la uva caimarona (Pourouma cecropiifolia) y el musgo (Bryophyta sensu stricto), o de espacios como la maloca, en cuyas grandes entradas nunca nadie se «tranca».

Un aspecto importante de este período es la necesidad femenina de reconocer una empatía masculina frente al manejo del parto, para lo cual ambos fueron formados desde la infancia. Es decir, no solo en el embarazo y posparto se da una participación del hombre alrededor de los cuidados, sino que durante el parto el padre también realiza actividades para proteger este momento de alta vulnerabilidad. Ello es evidente en dos relatos: el primero de una partera tikuna residente en el área periurbana, y el segundo de un hombre murui residente en el área rural: 
Yo le digo a mi marido, si yo tengo dolores nadie tiene que venir a verme, yo no llamo a nadie [...]. Si yo voy a sentir los dolores, él [el padre] debe sentirlos también, él es el que me atiende, mi partero.

Esas son cosas de mujeres. Nosotros los hombres no entramos porque nos podemos enfermar, nos da la saladera, pero desde afuera estamos también haciendo fuercita y cuidando con tabaco.

Finalmente, para la etapa en la que a la mujer «se le hace descansar» - es decir, el momento del nacimiento del bebé y el alumbramiento de la placenta-, los acompañantes o la madre ya tienen listos los remedios vegetales, mantas y ropa necesarios para evitar que a ella o al neonato les «entre el frío». Luego de la salida de la placenta, se hace el corte y amarre del cordón umbilical. Este manejo del cordón también depende de la procedencia étnica. Por ejemplo, en las etnias tikuna y cocama, el corte lo realiza el padrino, escogido según criterios étnicos y familiares - pues el padre o la madre podrían enfermar si lo hacen ellos mismos-y durante él se da el nombre. Lo anterior es explicado por un padre tikuna residente en área rural:

Yo, el papá del niño, si cortaba el ombligo, yo cometía una falta gravísima según las costumbres tikunas y cocamas, porque entonces yo me convertía en una runa mula, que es la transformación del hombre en una bestia, digamos un caballo; y si no pasaba eso, el peligro era mayor porque por ejemplo, iba a la selva y se encontraba con un mico grande que los tikunas lo llaman awara, que es el castigador de aquellas personas que cometían el adulterio, entonces es por ejemplo como si la comadre viviera con el compadre o la cuñada con el papá, es algo así de la misma sangre [...] por eso antes se escoge el padrino que tendrá una gran responsabilidad como cuidar a la comadre durante el tiempo de dieta, o cuando algo pasaba con los padres el padrino se hacía cargo de ese niño.

Este es uno de los aspectos que más difiere entre etnias. Por ejemplo, los cocamas realizan el ritual del ishpa durante el cual, padre y padrino sellan la alianza espiritual por medio del consumo de unas copas de licor, consideradas la orina del recién nacido que se incorpora en sus cuerpos convirtiéndolos en parientes, hoy en día adoptado por los pobladores tikuna de la región. En los pueblos murui y bora, la asignación del nombre que definirá su identidad se hace mediante una fiesta/baile realizado entre los tres y cuatro años de nacido, lo que coincide con el destete en zonas apartadas, pues en los cascos urbanos y comunidades periurbanas este se presenta en edades más tempranas. Un ejemplo de lo anterior se evidencia en el relato de abuela bora residente hoy en día en área urbana: 
Cuando el bebé nace le untan leche de siringa [caucho - Hevea brasiliensis] y con ella le pegan la lanita café que se ve adentro del nido de colibrí y que el papá ha recogido durante todo el embarazo. Con esa lanita el bebé parece un osito y durante un mes [la dieta] no se le quita y se bañan solo sus partecitas. Al mes se halan y el bebé llora. Por eso los boras no tenemos pelo [...] El nombre se le da cuando ya tiene tres o cuatro, en una fiesta.

Mientras el padre entierra la placenta en un lugar cercano o bajo la casa para adherir al nuevo ser al territorio-, la partera, familiar o la misma madre hace los baños del bebé y de ella, generalmente con algodón morado (Gossypium herbaceum L.) y ubo (Spondias mombin), para evitar complicaciones de la matriz y la incorporación de características negativas en el cuerpo del bebé.

La lactancia inicia después de que la madre y el bebé estén limpios y vestidos, lo cual ocurre en la primera hora o dos horas después del nacimiento. Lo anterior indica que, a diferencia de los períodos establecidos por el SBM para posparto, esta primera etapa de puerperio inmediato es concebida aún como parte constitutiva del parto, pues es precisamente la leche materna la encargada de humanizar a la criatura. Asimismo, al considerar la sangre como el elemento de mayor vulnerabilidad ante los seres no humanos del territorio, pues los hace visibles por su color, olor y limpieza, tal como sucede en sala de partos definirá el final de esta etapa.

\section{Dieta o posparto}

Esta es la etapa más variable y disímil frente a los períodos establecidos por el SBM. Se divide en: (i) puerperio inmediato (dos primeras horas); (ii) puerperio mediato (dos a 48 horas posparto); (iii) puerperio precoz (aproximadamente hasta los cuarenta días después del parto), y (iv) puerperio tardío (hasta los seis meses según MSPS, 2013, p. 63). En el SMT, se presentan variaciones que no obedecen exclusivamente a criterios étnicos, sino que se relacionan con nociones familiares, disponibilidad de tiempo para el cuidado y responsabilidades dentro y fuera del espacio doméstico. Al respecto se establecen tres etapas que permiten la generalización de los cuidados asociados a ellos en los diferentes pueblos amazónicos: (i) «dieta del toldillo o los nueve días»; (ii) la «criatura rojita», es decir, cargada de sangre, y (iii) el «bebé fuertecito» o puerperio tardío.

La etapa inicial «dieta del toldillo», también abordada en el trabajo de Santos (2018) con la comunidad tikuna de Arara, es descrita por la población como «los nueve días de guarde». Se encuentra como aspecto común la referencia al encierro de la madre y el recién nacido durante siete o nueve días, período durante el cual 
no deben salir del cuarto y la madre se dedica exclusivamente a lactar, recuperar fuerza, protegerse de los cambios de temperatura y alejarse, tanto ella como la criatura, de los seres no humanos atraídos por la sangre que brotó del parto.

El padre, por su parte, puede salir del cuarto, mas no de la casa, pues el encuentro con seres que cutipan presentes en la chagra y la selva puede enfermar al bebé o a la madre. Si por alguna razón debe salir de su hogar, tendrá que recurrir a un médico tradicional o chamán que amarre sus extremidades con tabaco para protegerlo de estos males o características no deseadas. Esta curación hoy en día es realizada desde el nacimiento, dadas las responsabilidades laborales del padre.

Para la segunda etapa, o «criatura rojita», los cuidados obedecen a una concepción de una criatura aún cargada de sangre y sin el espíritu asentado, aspectos que la hacen vulnerable a los espíritus o cutipas del entorno, cambios de temperatura, entrada de aire por las mollejas, entre otros, así como de los males cargados por los progenitores debido a que los tres comparten la misma sangre.

Esta noción fomenta una activa participación del padre y de la red familiar cercana dentro de la «couvade» o «covada», definida como el ritual de parentesco compartido o período de dieta de los progenitores. Esta se soporta en la idea de formación conjunta del recién nacido, que desde la gestación recibe las sustancias de madre y padre, y como tal, los tres se encuentran biológica, mental y espiritualmente entrelazados. Esto explica la necesidad de cuidado mutuo para evitar males que puedan tener un impacto negativo en la salud del nuevo ser (Belaunde, 2008, pp. 43-44).

Quizá durante esta etapa la enfermedad más reconocida y temida en gran cantidad de pueblos andino-amazónicos es el «sobreparto» ${ }^{6}$, que a pesar de no contar con un cuadro sintomático definido, presenta un gran conocimiento colectivo sobre su etiología. Se encuentra asociado a la falta de cuidados durante el posparto, ya sea por exposición a temperaturas frías o por esfuerzo físico excesivo. Para ello, la mayoría de remedios vegetales reportados se ciñen al acto de «sanar el vientre» después del parto con el fin de que la mujer pueda mantener la fuerza y seguir trabajando sin dolores o debilidad (Reyes, 2009a, p. 113).

En ese sentido, se recurre a diversos productos vegetales disponibles en su territorio (ver tabla 5), entre los que se destacan el algodón morado (Gossypium herbaceum L.), uña de gato (Uncaria guianensis y U. tomentosa), corteza de ubo (Spondias mombin) y socoba (Himatanthus sucuuba), tal como lo explica una partera tikuna residente en área rural:

6 «El sobreparto es un término utilizado para denominar al conjunto de síntomas de hemorragia, dolor pélvico y fiebre, que pueden atribuirse a la endometritis postparto» (Reyes, 2009a, p. 118). 
Se ponen a hervir corteza de socoba, ubo y cinco hojas de algodón morado. Esa agua se pone debajo de la hamaca donde está la mamá, y se le va secando el sudor que le da por el calor. Cuando el agua se enfría, la mamá se sienta en ella para hacerse el baño.

La finalización de esta etapa es muy variable, pero se encuentra alrededor del mes a los tres meses después del parto. No obstante, en los diferentes pueblos amazónicos coincide el uso de pinturas corporales como huito (Genipa americana), achiote (Bixa orellana) o carayurú (Arrabidaea chica), o prácticas rituales para la protección del cuerpo de la criatura al salir al nuevo contexto natural y social, afianzadas en el cuerpo del recién nacido por medio de pulseras o collares.

La tercera etapa, o «bebé fuertecito», inicia a partir del sostenimiento de la cabeza, capacidad de sentarse o salida de los primeros dientes, rasgos físicos que invitan a la ablactancia o alimentación complementaria, que suele iniciarse con el consumo de coladas de plátano y masato de yuca no fermentado. A partir de esta etapa, los ciclos de crecimiento y desarrollo del niño o niña se pautarán por sus cambios corporales y biológicos evidentes, más que por edades. Su finalización se da frente al evidente fortalecimiento de la caminata del bebé, momento en el que adquiere gran independencia y empieza a comer solo.

Tabla 5. Plantas medicinales empleadas en el posparto

\begin{tabular}{lllllll}
\hline $\begin{array}{l}\text { Nombre } \\
\text { común }\end{array}$ & $\begin{array}{c}\text { Nombre } \\
\text { científico }\end{array}$ & $\begin{array}{c}\text { Parte } \\
\text { utilizada }\end{array}$ & $\begin{array}{l}\text { Usos medi- } \\
\text { cinales }\end{array}$ & Preparación & $\begin{array}{c}\text { Adminis- } \\
\text { tración }\end{array}$ & Dosificación \\
\hline $\begin{array}{l}\text { Uña de } \\
\text { gato }\end{array}$ & $\begin{array}{l}\text { Uncaria } \\
\text { tomentosa/ } U . \\
\text { guianensis }\end{array}$ & Corteza & $\begin{array}{l}\text { Limpieza } \\
\text { de útero/ } \\
\text { Hemorra- } \\
\text { gias }\end{array}$ & Decocción & $\begin{array}{l}\text { Oral/ } \\
\text { baño }\end{array}$ & $\begin{array}{l}\text { Tomar varias veces } \\
\text { en el día hasta aca- } \\
\text { bar el remedio. Hacer } \\
\text { lavado vaginal }\end{array}$ \\
\hline $\begin{array}{l}\text { Renaco/ } \\
\text { Renaquillo }\end{array}$ & Clusia rosea & Corteza & $\begin{array}{l}\text { Limpieza } \\
\text { de útero }\end{array}$ & Maceración/ & Oral & $\begin{array}{l}\text { Tomar ocho días } \\
\text { después del parto }\end{array}$ \\
\hline Cilantrón & $\begin{array}{l}\text { Eryngium } \\
\text { foetidum }\end{array}$ & Hoja & $\begin{array}{l}\text { Limpieza } \\
\text { de útero }\end{array}$ & Decocción & Oral & $\begin{array}{l}\text { Tomar varias veces } \\
\text { en el día hasta sentir } \\
\text { la mejoría }\end{array}$ \\
\hline $\begin{array}{l}\text { Huaca- } \\
\text { purana }\end{array}$ & $\begin{array}{l}\text { Campsiandra } \\
\text { angustifolia }\end{array}$ & Corteza & $\begin{array}{l}\text { Limpieza } \\
\text { de útero }\end{array}$ & $\begin{array}{l}\text { Maceración/ } \\
\text { Decocción }\end{array}$ & Oral & Tomar tres veces \\
\hline $\begin{array}{l}\text { Chuchu- } \\
\text { huasa }\end{array}$ & $\begin{array}{l}\text { Maytenus } \\
\text { laevis }\end{array}$ & Corteza & $\begin{array}{l}\text { Limpieza } \\
\text { de útero }\end{array}$ & $\begin{array}{l}\text { Maceración/ } \\
\text { Decocción }\end{array}$ & Oral & $\begin{array}{l}\text { Tomar en las } \\
\text { mañanas }\end{array}$ \\
\hline $\begin{array}{l}\text { Palo } \\
\text { sangre }\end{array}$ & $\begin{array}{l}\text { Brosimum } \\
\text { rubescens }\end{array}$ & Corteza & $\begin{array}{l}\text { Limpieza } \\
\text { de útero }\end{array}$ & $\begin{array}{l}\text { Decocción/ } \\
\text { Mezcla con } \\
\text { otras plantas }\end{array}$ & Oral & $\begin{array}{l}\text { Tomar varias veces al } \\
\text { día por un mes }\end{array}$ \\
\hline Mango & $\begin{array}{l}\text { Mangifera } \\
\text { indica L. }\end{array}$ & Corteza & $\begin{array}{l}\text { Limpieza } \\
\text { de útero }\end{array}$ & $\begin{array}{l}\text { Decocción/ } \\
\text { Mezcla con } \\
\text { otras plantas }\end{array}$ & Oral & $\begin{array}{l}\text { Tomar varias veces al } \\
\text { día por un mes }\end{array}$ \\
\hline
\end{tabular}




\begin{tabular}{|c|c|c|c|c|c|c|}
\hline $\begin{array}{l}\text { Nombre } \\
\text { común }\end{array}$ & $\begin{array}{l}\text { Nombre } \\
\text { científico }\end{array}$ & $\begin{array}{c}\text { Parte } \\
\text { utilizada }\end{array}$ & $\begin{array}{l}\text { Usos medi- } \\
\text { cinales }\end{array}$ & Preparación & $\begin{array}{l}\text { Adminis- } \\
\text { tración }\end{array}$ & Dosificación \\
\hline Guanabana & $\begin{array}{l}\text { Annona } \\
\text { muricata }\end{array}$ & Hoja & $\begin{array}{l}\text { Limpieza } \\
\text { de útero }\end{array}$ & Decocción & Oral & $\begin{array}{l}\text { Tomar varias veces } \\
\text { en el día hasta sentir } \\
\text { la mejoría }\end{array}$ \\
\hline Malva & $\begin{array}{l}\text { Malva } \\
\text { silvestris }\end{array}$ & Hoja & $\begin{array}{l}\text { Limpieza } \\
\text { de útero }\end{array}$ & $\begin{array}{l}\text { Decocción/ } \\
\text { Mezcla con } \\
\text { otras plantas }\end{array}$ & $\begin{array}{l}\text { Oral/ } \\
\text { baño }\end{array}$ & $\begin{array}{l}\text { Tomar varias veces en } \\
\text { el día. Pasada media } \\
\text { hora del parto se sienta } \\
\text { la mujer en el agua }\end{array}$ \\
\hline Ubo & $\begin{array}{l}\text { Spondias } \\
\text { mombin }\end{array}$ & Corteza & $\begin{array}{l}\text { Limpieza } \\
\text { de útero/ } \\
\text { Sobreparto }\end{array}$ & $\begin{array}{l}\text { Decocción/ } \\
\text { Mezcla con } \\
\text { otras plantas }\end{array}$ & $\begin{array}{l}\text { Oral/ } \\
\text { vapori- } \\
\text { zación }\end{array}$ & $\begin{array}{l}\text { Tomar dos veces } \\
\text { durante } 15 \text { días }\end{array}$ \\
\hline Socoba & $\begin{array}{l}\text { Himatanthus } \\
\text { sucuuba }\end{array}$ & Hoja & Sobreparto & $\begin{array}{l}\text { Decocción/ } \\
\text { Mezcla con } \\
\text { otras plantas }\end{array}$ & $\begin{array}{l}\text { Vapori- } \\
\text { zación }\end{array}$ & $\begin{array}{l}\text { Se combina la corteza } \\
\text { con } 5 \text { hojas de flor de } \\
\text { algodón y } 5 \text { hojas de } \\
\text { socoba. La mujer se le } \\
\text { pone a «humear» hasta } \\
\text { que el agua se enfría y } \\
\text { se baña con esa agua }\end{array}$ \\
\hline $\begin{array}{l}\text { Hierba } \\
\text { luisa }\end{array}$ & $\begin{array}{l}\text { Aloysia } \\
\text { citriodora }\end{array}$ & Hoja & Sobreparto & Decocción & Oral & $\begin{array}{l}\text { Tomar varias veces } \\
\text { en el día }\end{array}$ \\
\hline Jengibre & $\begin{array}{l}\text { Zingiber } \\
\text { officinale } \\
\text { Roscoe }\end{array}$ & Rizoma & Sobreparto & Decocción & Oral & $\begin{array}{l}\text { Tomar varias veces } \\
\text { en el día hasta acabar } \\
\text { el remedio }\end{array}$ \\
\hline $\begin{array}{l}\text { Algodón } \\
\text { morado }\end{array}$ & $\begin{array}{l}\text { Gossypium } \\
\text { herbaceum L. }\end{array}$ & Hoja & Sobreparto & $\begin{array}{l}\text { Decocción/ } \\
\text { Mezcla con } \\
\text { otras plantas }\end{array}$ & $\begin{array}{l}\text { Oral/ } \\
\text { Baño }\end{array}$ & $\begin{array}{l}\text { Tomar varias veces } \\
\text { en el día. Después } \\
\text { del parto se sienta la } \\
\text { mujer en el agua }\end{array}$ \\
\hline Ojé & Ficus insipida & Resina & Lactancia & $\begin{array}{l}\text { Mezcla con } \\
\text { azúcar }\end{array}$ & Oral & Una toma \\
\hline Plátano & $\begin{array}{l}\text { Musa } \\
\text { paradisiaca }\end{array}$ & Fruto & Lactancia & $\begin{array}{l}\text { Maceración/ } \\
\text { Decocción }\end{array}$ & Oral & $\begin{array}{l}\text { Tomar como colada } \\
\text { una vez al día }\end{array}$ \\
\hline Yuca & $\begin{array}{l}\text { Manihot } \\
\text { esculenta }\end{array}$ & $\begin{array}{l}\text { Tubér- } \\
\text { culo }\end{array}$ & Lactancia & $\begin{array}{l}\text { Maceración/ } \\
\text { Decocción }\end{array}$ & Oral & $\begin{array}{l}\text { Tomar el almidón } \\
\text { varias veces en el día }\end{array}$ \\
\hline Ortiga & Urtica dioica & Flor & Lactancia & Decocción & Oral & $\begin{array}{l}\text { Frotar el agua en el } \\
\text { seno con la flor }\end{array}$ \\
\hline Caimo & $\begin{array}{l}\text { Chryso- } \\
\text { phyllum cainito }\end{array}$ & Hoja & Lactancia & $\begin{array}{l}\text { Decocción/ } \\
\text { Mezcla con } \\
\text { otras plantas }\end{array}$ & Baño & $\begin{array}{l}\text { Coger un peine y } \\
\text { «peinar» el seno con } \\
\text { el agua }\end{array}$ \\
\hline Papaya & Carica papaya & Hoja & Lactancia & $\begin{array}{l}\text { Decocción/ } \\
\text { Mezcla con } \\
\text { otras plantas }\end{array}$ & Baño & $\begin{array}{l}\text { Tomar varias veces } \\
\text { en el día }\end{array}$ \\
\hline $\begin{array}{l}\text { Rosa Sisa/ } \\
\text { Flor de } \\
\text { difunto }\end{array}$ & Tagetes erecta & Hoja & $\begin{array}{l}\text { No le entre } \\
\text { al niño mal } \\
\text { de aire }\end{array}$ & Decocción & Baño & $\begin{array}{l}\text { Se baña al recién } \\
\text { nacido una sola vez }\end{array}$ \\
\hline
\end{tabular}




\section{DISCUSIÓN}

La información recolectada permite concluir que las prácticas de atención y cuidado a la salud reproductiva femenina se encuentran determinadas por cuatro categorías de comprensión que permiten la generalización de los resultados en la mayoría de los pueblos indígenas del territorio. Estas son: (i) «formación de personas»; (ii) «construcción de los cuerpos» (fuerza, consustancialidad y regulación de la temperatura), y (iii) buen trato. La primera de ellas, «formación de personas», hace referencia a la formación de personas saludables y adaptables al contexto, cuya brújula moral le permita mantener buenas relaciones sociales y familiares, y por ello, una buena salud.

Las destrezas necesarias para manejar el hogar y criar a los hijos se trasmiten en las actividades cotidianas y las narrativas familiares (generalmente de abuelos y abuelas), entrelazadas con un conocimiento ritual y mitológico no explícito, pero que reflejan que la producción de gente y el cuidado de su salud y que dependen de la armonización de las relaciones entre los diferentes seres, sustancias y fuerzas que componen el territorio (Oenning da Silva, 2015, p. 24).

Lo anterior se presenta en el marco de una sociedad que concibe la fertilidad como sinónimo de salud y bienestar, pues los hijos e hijas representan la alegría de un hogar y su cuidado se encuentra bajo la corresponsabilidad de una familia extendida que no solo promueve los lazos de consanguinidad y afinidad conducente al tejido de una amplia red de apoyo, sino que otorga un reconocimiento social a los valores asociados a la maternidad. Esto se convierte en un factor protector de la salud mental materna que, al no concebir la crianza como una dificultad, hace de los cuidados perinatales un ritual cotidiano para la consecución de la buena vida en comunidad.

La segunda de estas categorías, «construcción de los cuerpos», se soporta en la premisa de la construcción colectiva del cuerpo, cuya forma y comportamiento biológico se definen mediante su relación con otros cuerpos y con el territorio ambiental y social (Reyes, 2009b, p. 207). En esta categoría encontramos la formación - desde la concepción - de cuerpos fuertes, con gran resistencia, que contrastan con una sociedad «occidental» algiofóbica que ofrece analgésicos ante el menor asomo de dolor. Estos cuerpos, además, son disciplinados y con capacidad de controlar su vulnerabilidad en relación con el territorio, por medio de prácticas cotidianas de alimentación, dieta, reclusión, curaciones y rituales, que demuestran la fluidez de un cuerpo que altera su estado - $\mathrm{y}$ el de la persona - mediante el flujo constante de sustancias, fuerzas, humores o seres que lo modifican (Oenning da Silva, 2015, p. 24). 
En esta etapa encontramos la «consustancialidad» o incorporación en los cuerpos de las propiedades de un elemento vegetal o animal por contacto directo o indirecto. Acá se reconocen términos como la «cutipa», asociada a características que deben ser evitadas, y las «curaciones», como la búsqueda de incorporación de características deseables. Ello demuestra no solo el gran conocimiento que tienen estas comunidades frente a la medicina vegetal y los animales que las rodean, sino también el mantenimiento de las teorías nativas sobre la hidráulica sanguínea y la hematología amazónica (Belaunde, 2008), en las que el manejo de la sangre corporal de los progenitores determina no solo el bienestar del recién nacido, sino la reproducción del cuerpo social, es decir, de la comunidad. Esto es evidente en el extendido temor de reproducirse con seres no humanos, como en el caso del delfín rosado (Inia geoffrensis) o bugeo, y el mal de lupuna (Ceiba pentandra), que pueden generar criaturas mixtas cuyos cuerpos no tendrán futuro en el mundo de la humanidad y, por ello, deben ser expulsados del espacio humano (Gow, 1997, p. 48).

También encontramos la «regulación de la temperatura corporal» de la parturienta y puérpera, pues durante la gestación la mujer se encuentra en un estado de calor. Su cuerpo, al abrirse por causa del parto, entra en un período de frío, tanto por la pérdida de sangre que mantiene caliente el cuerpo como por la apertura de los poros, la cabeza y el vientre (Acosta, Alegría, Cajiao, Llano, Valencia y Zuluaga, 1997, p. 45; Argote, Bejarano, Ruiz de Cárdenas, Muñoz de Rodríguez y Vásquez, 2004, p. 28; Sadler, Obach, Montecino, Naranjo, Martínez, Quiñelén y Quiñelén, 2006, p. 24 a 30). En ese sentido, el aire que enfría la sangre se convierte en un factor considerable de riesgo para la salud de la madre y por ello se restringen las actividades que impliquen cambios de temperatura (como planchar, lavar, cocinar, ventear la escoba o coger cuchillo), que pueden secar la leche, generar «resfrío de la matriz» o incorporar cualidades no deseadas al recién nacido.

Por último, encontramos el buen trato o «trato bonito», relacionado con la necesidad de generar un entorno de confianza, intimidad y trato respetuoso con el fin de garantizar la seguridad del parto y el nacimiento, así como un temperamento tranquilo en el recién nacido. Ello coincide con las disposiciones de la Organización Mundial de la Salud (OMS) frente a la atención al parto normal (Chalmers, Mangiaterra y Porter, 2002, pp. 136 y 137), que reconocen la importancia de generar un ambiente cálido y tranquilo para reducir los factores de riesgo de complicaciones obstétricas. Quizá esto, sumado a la cercanía geográfica, familiar y cultural, sea la razón principal de la evidente preferencia frente a la atención 
por agentes del SMT para el cuidado de la salud reproductiva femenina, pues un entorno familiar y privado promueve la confianza en la mujer, que en ocasiones manifiesta pudor frente a personas desconocidas (como médicos, enfermeros, etc.) por considerar la sexualidad y el parto eventos íntimos (Laza, 2012, p. 55).

Desde esta óptica, el nacimiento de nuevas personas constituye un momento de trascendencia en la vida social, con una carga psicológica y afectiva considerable. Por lo tanto, estas categorías de comprensión definen de manera general las prácticas de cuidado y atención en las etapas de fertilidad, embarazo, parto y dieta en los diferentes pueblos indígenas participantes; además, muestran la concepción del nacimiento como un hecho universal que, a pesar de sus generalidades fisiológicas, es moldeado e interpretado por cada cultura y, de acuerdo con ello, se determina la forma de entenderlo, asistirlo o acompañarlo.

Es por ello que en la Amazonia colombiana se hace necesaria la articulación práctica de los modelos de salud presentes en el territorio, dada su diversidad étnica-cultural y pluralismo médico de gran incidencia en las pautas de cuidados en salud de la población. Dicho pluralismo obliga a los sistemas de salud a generar nuevas estrategias para que la identidad étnica y cultural de los usuarios y usuarias no se constituyan en una barrera de acceso y calidad en la atención (Alarcón et al., 2003, p. 1062).

Para ello se requieren competencias culturales que den pie a espacios sensibles, de respeto mutuo, con conciencia de sus propias creencias, conocimientos de las diferencias culturales y un uso apropiado del lenguaje (Martínez et al., 2006, p. 340). Por tanto, se reconoce la necesidad de abrir el diálogo con los agentes del SMT, conocer su cultura médica, aceptar su legitimidad, y finalmente, reconocer las áreas de conflicto y acuerdo. De esta forma, se puede establecer un consenso que facilite la conformación de un modelo intercultural de atención a las etapas reproductivas de la mujer.

\section{REFERENCIAS}

Acosta, Mónica, Liliana Alegría, Gloria Estella Cajiao, Ana Milena Llano, Celmira Valencia y Patricia Zuluaga (1997). Creencias populares sobre el autocuidado durante el puerperio, en las instituciones de salud de nivel 1. Colombia Médica, $8(1), 42-50$.

Alarcón, Ana, Aldo Vidal y Jaime Neira (2003). Salud intercultural: elementos para la construcción de sus bases conceptuales. Revista Médica de Chile, 131, 10611065. https://doi.org/10.4067/S0034-98872003000900014 
Argote, Luz, Nancy Bejarano, Carmen Ruiz de Cárdenas, Lucy Muñoz de Rodríguez y Martha Vásquez (2004). Transitando la adolescente por el puerperio: amenazas, peligros y acciones de protección durante la dieta. Aquichan, 4(1), 18-29.

Belaunde, Luisa Elvira (2008). El recuerdo de luna: género, sangre y memoria entre los pueblos amazónicos. Lima: Fondo Editorial de la Facultad de Ciencias Sociales, Universidad Nacional Mayor de San Marcos, Centro Amazónico de Antropología y Aplicación Práctica (CAAAP).

Campos, Jaime (2001). Proyecto Andino-Europeo de Telemedicina para la Amazonia. Revista de Salud Pública: Terceras Jornadas Internacionales Médicas de la Amazonia, 3(1), 173-178.

Chalmers, B., V. Mangiaterra y R. Porter (2002). Principios de la Organización Mundial de la Salud (OMS) acerca del cuidado perinatal: guía esencial para el cuidado antenatal, perinatal y postparto. Revista del Hospital Materno Infantil Ramón Sardá, 21(3), 136-139.

Gow, Peter. (1997). O parentesco como consciencia humana: o caso dos piro. MANA, 3(2), 39-65. https://doi.org/10.1590/S0104-93131997000200002

Lagos, Carolina (2015). Plantas medicinales utilizadas en el tratamiento de enfermedades ginecológicas en Leticia y Puerto Nariño (Amazonas, Colombia). Etnobiología, 13(1), 53-72.

Laza, Celmira (2012). Una aproximación al estado de la partería tradicional en Colombia. Index de Enfermería, 21(1-2), 53-57. https://doi.org/10.4321/S113212962012000100012

Martínez, Manuel Francisco, Julia Martínez y Visitación Calzado (2006). La competencia cultural como referente de la diversidad humana en la prestación de servicios y la intervención social. Psychosocial Intervention, 15(3), 331-350. https://doi. org/10.4321/S1132-05592006000300007

Ministerio de Salud del Perú (2006). Análisis de la situación de salud del pueblo achuar. Serie análisis de situación de salud y tendencias. Perú: Dirección Nacional de Epidemiología.

Ministerio de Salud y Protección Social - MSPS (2013). Análisis de situación de salud según regiones Colombia. Bogotá: Dirección de Epidemiología y Demografía Grupo ASIS.

Ministerio de Salud y Protección Social - MSPS (2014). Análisis de situación de salud. Colombia, 2013. Bogotá: Ministerio de Salud y Protección Social.

Ministerio de Salud y Protección Social - MSPS y Fondo de Población de las Naciones Unidas - UNFPA (2014). Protocolos para la atención de enfermería a la salud sexual y reproductiva de la mujer. Principios y consideraciones generales; 
Cuidado de la mujer en edad fértil; Cuidado de la mujer gestante; Cuidado de la mujer en trabajo de parto, parto y puerperio. Bogotá D.C.: MSPS y UNFPA

Oenning da Silva, Rita de Cácia (2015). La educación de sí mismo y la producción del niño indígena en el noroeste amazónico de Brasil. Anthropologica, 33(35), $15-40$.

Patiño, Ana Eugenia y María Sandín (2014). Diálogo y respeto: bases para la construcción de un sistema de salud intercultural para las comunidades indígenas de Puerto Nariño, Amazonas, Colombia. Salud Colectiva, 10(3), 379-396. https:// doi.org/10.18294/sc.2014.400

Patton, M. (2002). Qualitative research and evaluation methods. Tercera edición. Thousand Oaks: Sage Publications.

Profamilia, MSPS, Bienestar Familiar y USAID (2011). Encuesta Nacional de Demografia y Salud ENDS 2010. Colombia: Profamilia, Ministerio de la Protección Social (MPS), Instituto Colombiano de Bienestar Familiar (ICBF) y Agencia de los Estados Unidos para el Desarrollo Internacional (USAID).

Reyes, Giovanna (2009a). Analogías y antagonismos en salud sexual y reproductiva entre población tikuna y los servicios de salud del municipio de Leticia. Tesis para optar por el título de magister en Estudios Amazónicos. Leticia, Colombia: Universidad Nacional de Colombia Sede Amazonia.

Reyes, Giovanna (2009b). El cuerpo como unidad biológica y social: una premisa para la salud sexual y reproductiva. Revista Colombiana de Antropología, 45(1), 203-224

Rivas, R. (2015). Prácticas de crianza en niños y niñas menores de tres años en los pueblos tikuna, yagua y kukama. Lima: Ministerio de Educación.

Sadler, Michelle, Alexandra Obach, Sonia Montecino, Carola Naranjo, Rosa Martínez, María Quiñelén y Laura Quiñelén (2006). Pautas de crianza mapuche. Estudio «Significaciones, actitudes y prácticas de familias mapuches en relación a la crianza y cuidado infantil de los niños desde la gestación hasta los cinco años». Chile: Centro Interdisciplinario de Estudios de Género CIEG Facultad de Ciencias Sociales Universidad de Chile, Departamento de Salud Pública CIGES, Universidad de la Frontera.

Santos, C. (2018). Pautas y prácticas de crianza contemporáneas de los niños tikuna de la comunidad de Arara. Tesis de Maestría en Estudios Amazónicos. Leticia: Universidad Nacional de Colombia Sede Amazonia. 